\title{
Gluon propagator with dynamical quarks
}

\author{
Joannis Papavassiliou* \\ Department of Theoretical Physics and IFIC, \\ University of Valencia and CSIC, \\ E-46100, Valencia, Spain. \\ E-mail: Joannis.Papavassiliou@uv.es
}

We review recent work on the effects of quark loops on the gluon propagator in the Landau gauge, relying mainly on the Schwinger-Dyson equations that describe the two-point sector of QCD. Particularly important in this context is the detailed study of how the standard gluon mass generation mechanism, which is responsible for the infrared finiteness of the quenched gluon propagator, is affected by the inclusions of dynamical quarks. This issue is especially relevant and timely, given the qualitative picture that emerges from recent unquenched lattice simulations. Our results demonstrate clearly that the gluon mass generation persists, and that the corresponding saturation points of the unquenched gluon propagators are progressively suppressed, as the number of quark flavors increases.

QCD-TNT-III-From quarks and gluons to hadronic matter: A bridge too far?, 2-6 September, 2013

European Centre for Theoretical Studies in Nuclear Physics and Related Areas (ECT*), Villazzano, Trento (Italy)

${ }^{*}$ Speaker. 


\section{Introduction}

In recent years outstanding progress has been made in our understanding of various aspects of the nonperturbative dynamics of Yang-Mills theories, through the fruitful combination of a variety of approaches and techniques [1, 2, 3, 4, 5, 6, 7, 8, 9, 10, 11]. In fact, we appear to have obtained a rather firm grasp on the infrared behavior of the fundamental Green's (correlation) functions of QCD, such as gluon, ghost, and quark propagators, as well as some of the basic vertices of the theory. Of course, the gluon propagator occupies deservedly a prominent position in this ongoing endeavor, because its infrared behavior is intimately connected to the fundamental question of if and how Yang-Mills theories generate dynamically a mass scale.

The full gluon propagator in the Landau gauge assumes the general form

$$
i \Delta_{\mu v}(q)=-i \Delta\left(q^{2}\right) P_{\mu v}(q) ; \quad P_{\mu v}(q)=g_{\mu v}-q_{\mu} q_{v} / q^{2},
$$

where $\Delta\left(q^{2}\right)$ is related to the scalar form factor of the gluon self-energy $\Pi_{\mu v}(q)=\Pi\left(q^{2}\right) P_{\mu v}(q)$ through

$$
\Delta^{-1}\left(q^{2}\right)=q^{2}+i \Pi\left(q^{2}\right) .
$$

As has been demonstrated in detail $[12,13]$ within the general framework of the SchwingerDyson equations (SDEs) [14], the nonperturbative dynamics of pure Yang-Mills theories gives rise to a dynamical (momentum-dependent) gluon mass [15, 16, 17], which accounts for the infrared finiteness of the quenched $\Delta\left(q^{2}\right)$, observed in large-volume lattice simulations, both in $S U(2)$ [6] and in $S U(3)$ [7]. In particular, in Minkowski space, the gluon propagator may be described in terms of two basic functions, $J\left(q^{2}\right)$ and $m^{2}\left(q^{2}\right)$

$$
\Delta^{-1}\left(q^{2}\right)=q^{2} J\left(q^{2}\right)-m^{2}\left(q^{2}\right),
$$

where, in the limit $q^{2} \rightarrow 0$, we have that $q^{2} J\left(q^{2}\right) \rightarrow 0$, whereas $m^{2}(0) \neq 0$. This property of the mass function $m^{2}\left(q^{2}\right)$ accounts for the fact that $\Delta\left(q^{2}\right)$ saturates at a non-vanishing constant value in the deep infrared $[2,15]$. The presence of this effective mass explains, in addition, the finiteness of the dressing function, $F\left(q^{2}\right)[2,3]$, which is related to the full ghost propagator, $D\left(q^{2}\right)$, by

$$
D\left(q^{2}\right)=\frac{F\left(q^{2}\right)}{q^{2}} .
$$

It is interesting to point out that, in $d=4$, the function $J\left(q^{2}\right)$ diverges logarithmically in the deep infrared, due to the fact that the ghost-loop contributing to it contains massless ghost propagators [18].

It turns out that new lattice simulations involving dynamical quarks [19] furnish further valuable information that permits us to scrutinize in much more detail the general dynamical scenario described above. In particular, the unquenched gluon propagators continue to saturate in the infrared, which suggests that the mass generation mechanism persists in the presence of quark loops. In fact, the observed considerable suppression of the value of their saturation points compared to the quenched ones clearly suggests that the corresponding gluon masses increase.

The purpose of this presentation is to report on recent work in the continuum, which fully confirms the general trends displayed by the lattice results of [19]. This particular study has been 
carried out within the framework provided by the synthesis of the pinch technique (PT) $[15,20$, 21, 22] with the background field method (BFM) [23], known in the literature as the PT-BFM scheme [1]. For a related analysis, see [24]. Note also that in the context of the so-called "scaling" solutions [4] the unquenching effects have been considered in [25, 26].

The general philosophy underlying the work of [27] may be summarized as follows. The momentum evolution of $m^{2}\left(q^{2}\right)$ is described by a homogeneous integral equation, to be referred to as the mass equation, whose kernel depends on $\Delta\left(q^{2}\right)$ in a complicated way. The detailed numerical study of this particular equation, for pure $S U(3)$ Yang-Mills, revealed that its solutions depend strongly on the precise shape of $\Delta\left(q^{2}\right)$ through a wide range of momenta [13]. Of course, the inclusion of dynamical quarks modifies the form of the gluon propagator, to be denoted by $\Delta_{N_{f}}\left(q^{2}\right)$; the modifications depend, among other things, on the number of quark families, $N_{f}$, and the values of the corresponding quark masses. In the context of the SDEs, the behavior of $\Delta_{N_{f}}\left(q^{2}\right)$ in the range of intermediate momenta may be obtained from the quenched gluon propagator $\Delta\left(q^{2}\right)$ by means of an approximate procedure, which attributes the main bulk of the "unquenching" to the fully dressed quark-loop graph, while higher loop contributions are considered to be subleading [28]. The value of $\Delta_{N_{f}}(0)$, however, is determined only in conjunction with the gluon mass equation, in whose kernel one must implement the change $\Delta\left(q^{2}\right) \rightarrow \Delta_{N_{f}}\left(q^{2}\right)$. Thus, the study presented here finally boils down to the simultaneous solution of the mass equation and the master formula that controls the amount by which $\Delta_{N_{f}}\left(q^{2}\right)$ deviates from the quenched $\Delta\left(q^{2}\right)$; for the latter we will use directly the lattice data of [7].

\section{Gluon mass generation in a nutshell.}

The gauge invariant generation of a gluon mass [15] proceeds through the implementation of the Schwinger mechanism [29, 30], which requires the existence of a very special type of nonperturbative vertices [31, 32, 33, 34, 35], which within the PT-BFM framework are generically denoted by $\widetilde{V}$. In particular:

(i) The $\widetilde{V}$ vertices are longitudinally coupled, and contain massless poles, which make possible that the SDE of the gluon propagator yields $\Delta^{-1}(0) \neq 0$.

(ii) The aforementioned poles have nonperturbative origin: they are colored composite states with vanishing mass. They act as Nambu-Goldstone bosons, maintaining gauge invariance, but, are not associated with the spontaneous breaking of any continuous symmetry. In particular, their presence guarantees that the Ward identities and the Slavnov-Taylor identities of the theory remain intact, i.e., they have the same form before and after mass generation.

(iii) These longitudinally coupled states decouple from on-shell amplitudes, and, in general, from physical observables.

The exact application of these notions at the level of the gluon propagator SDE is particularly subtle, and has been discussed in great detail in the recent literature $[12,13]$. The final upshot is the derivation of a homogeneous integral equation, valid only in the Landau gauge, which controls the momentum evolution of the gluon mass. It is given by [13] (see also Fig. 1)

$$
m^{2}\left(q^{2}\right)=-\frac{g^{2} C_{A}}{1+G\left(q^{2}\right)} \frac{1}{q^{2}} \int_{k} m^{2}\left(k^{2}\right) \Delta_{\rho}^{\mu}(k) \Delta^{v \rho}(k+q) \mathscr{K}_{\mu v}(k, q),
$$




$$
m^{2}\left(q^{2}\right)=\frac{1}{1+G\left(q^{2}\right)} \frac{1}{q^{2}} q^{\mu} \times \underbrace{2}_{1}
$$

Figure 1: The effective SDE satisfied by the dynamical gluon mass. The blue circle denotes the (conventional) fully-dressed three-gluon vertex $\Gamma_{\sigma \rho \beta}$, while the (red) $\widetilde{V}$ indicates a pole vertex whose external leg (with the little circled attached to it) is a background gluon. Finally, the perforated box marks the $Y\left(k^{2}\right)$, which represents the purely two-loop dressed correction to the one-loop dressed mass equation kernel.

with

$$
\begin{aligned}
\mathscr{K}_{\mu v}(k, q) & =\left[(k+q)^{2}-k^{2}\right]\{1-[Y(k+q)+Y(k)]\} g_{\mu v} \\
& +[Y(k+q)-Y(k)]\left(q^{2} g_{\mu v}-2 q_{\mu} q_{v}\right) .
\end{aligned}
$$

The quantity $Y$ corresponds to the subdiagram nested inside the two-loop dressed graph of Fig. 1, given by

$$
Y\left(k^{2}\right)=\frac{g^{2} C_{A}}{4 k^{2}} k_{\alpha} \int_{\ell} \Delta^{\alpha \rho}(\ell) \Delta^{\beta \sigma}(\ell+k) \Gamma_{\sigma \rho \beta}(-\ell-k, \ell, k),
$$

with $\Gamma_{\sigma \rho \beta}$ the full three-gluon vertex, and $C_{A}$ the Casimir eigenvalue in the adjoint representation $\left[C_{A}=N\right.$ for $\left.S U(N)\right]$. In the above equations we used the short-hand notation $\int_{k}=\mu^{\varepsilon} \int \mathrm{d}^{d} k /(2 \pi)^{d}$ to denote the dimensional regularization measure, where $d=4-\varepsilon$ is the space-time dimension and $\mu$ the 't Hooft mass.

Finally, the function $G\left(q^{2}\right)$ corresponds to the $g_{\mu \nu}$ component of a special two point-function, which constitutes a key ingredient in a set of powerful identities, relating the conventional Green's functions to those of the BFM $[22,36]$. In particular, for the case of the conventional gluon propagator, $\Delta$, and the PT-BFM gluon propagator, denoted in the literature by $\widehat{\Delta}$, the corresponding identity reads

$$
\Delta\left(q^{2}\right)=\left[1+G\left(q^{2}\right)\right]^{2} \widehat{\Delta}\left(q^{2}\right) ;
$$

its application at the level of the gluon SDE gives rise to the factor $1+G\left(q^{2}\right)$ in Eq. (2.1).

Note that, in the Landau gauge only, the quantity $1+G\left(q^{2}\right)$ is linked to the inverse of the ghost dressing function $F\left(q^{2}\right)$ through [22,37]

$$
F^{-1}\left(q^{2}\right) \approx 1+G\left(q^{2}\right)
$$

This relation, which is valid to a very good approximation, and becomes an exact equality at $q^{2}=0$, allows one to use the lattice results of [7] for the ghost dressing function, in order to determine $G\left(q^{2}\right)$.

It is obvious that the function $Y\left(k^{2}\right)$ represents a crucial ingredient of Eq. (2.1). However, its exact closed form is not available, mainly because our present knowledge of the full three-gluon vertex, entering in its definition, is incomplete (for recent studies see [5, 38]). We must therefore resort to approximate expressions for this quantity. In particular, we will employ the lowest-order 
perturbative expression for $Y\left(k^{2}\right)$, obtained from Eq. (2.3) by substituting the tree-level values for all quantities appearing there. Within this approximation, and after carrying out momentum subtraction renormalization (MOM) at $k^{2}=\mu^{2}$, one finds [13]

$$
Y_{\mathrm{R}}\left(k^{2}\right)=-\frac{\alpha_{s} C_{A}}{4 \pi} \frac{15}{16} \log \frac{k^{2}}{\mu^{2}},
$$

where $\alpha_{s}$ is the value of the coupling at the subtraction point chosen. This simple approximation will be compensated, in part, by multiplying $Y_{\mathrm{R}}\left(k^{2}\right)$ by and arbitrary constant $C$, i.e., by implementing the replacement $Y_{\mathrm{R}}\left(k^{2}\right) \rightarrow C Y_{\mathrm{R}}\left(k^{2}\right)$ and treating $C$ as a free parameter. In this heuristic way, one hopes to model further corrections that may be added to the "skeleton" result provided by Eq. (2.6). For a more sophisticated analysis based on the renormalization group properties of Eq. (2.1), see [39].

\section{The unquenching formula: getting $\Delta_{N_{f}}\left(q^{2}\right)$ from $\Delta\left(q^{2}\right)$}

As has been explained in [28], when the number of quark families is relatively small, it is reasonable to assume that the main bulk of the unquenching effects is captured by the (fully dressed) one-loop diagram of Fig. 2 neglecting, at this level of approximation, all contributions stemming from (higher order) diagrams containing nested quark loops.

Let us now turn to the form of this particular quark-loop diagram within the PT-BFM scheme. Factoring out the trivial color structure $\delta^{a b}$, we obtain

$$
\widehat{X}^{\mu v}\left(q^{2}\right)=-g^{2} d_{f} \int_{k} \operatorname{Tr}\left[\gamma^{\mu} S(k) \widehat{\Gamma}^{v}(k+q,-k,-q) S(k+q)\right],
$$

where $d_{f}$ is the Dynkin index of the fundamental representation $\left[d_{f}=1 / 2\right.$ for $\left.S U(3)\right]$, and $S$ denotes the full quark propagator, where, in the usual notation,

$$
S^{-1}(p)=-i[A(p) \not p-B(p)]=-i A(p)[\not p-\mathscr{M}(p)],
$$

and the ratio $\mathscr{M}(p)=B(p) / A(p)$ is the dynamical quark mass. The vertex $\widehat{\Gamma}_{\mu}$ corresponds to the PT-BFM quark-gluon vertex, satisfying the QED-like Ward identity [22]

$$
i q^{\mu} \widehat{\Gamma}_{\mu}(k,-k-q, q)=S^{-1}(k)-S^{-1}(k+q) .
$$

As a consequence of this Ward identity, it is immediate to show that

$$
q^{\mu} \widehat{X}_{\mu v}(q)=0
$$

a fact that allows one to cast $\widehat{X}_{\mu \nu}$ into the form

$$
\widehat{X}_{\mu v}(q)=\widehat{X}\left(q^{2}\right) P_{\mu v}(q) .
$$

Then, taking the trace $\widehat{X}_{\mu}^{\mu}(q)$, we obtain from Eq. (3.1) the expression

$$
\widehat{X}\left(q^{2}\right)=-\frac{g^{2}}{6} \int_{k} \operatorname{Tr}\left[\gamma^{\mu} S(k) \widehat{\Gamma}_{\mu}(k,-k-q, q) S(k+q)\right] .
$$




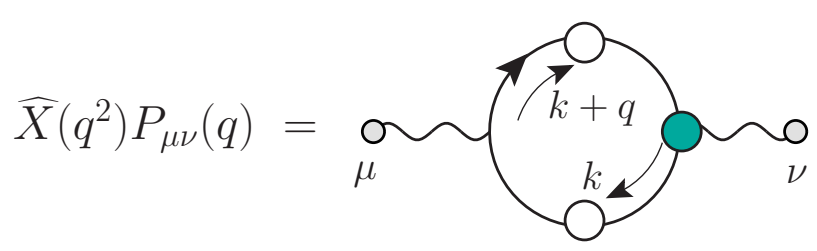

Figure 2: Diagrammatic representation of the nonperturbative quark loop, $\widehat{X}_{\mu \nu}$, which determines, at this level of approximation, the unquenching effects. The fully-dressed (green) vertex represents the PT-BFM vertex $\widehat{\Gamma}_{\mu}$.

The simple WI satisfied by the vertex $\widehat{\Gamma}_{\mu}$ turns out to be particularly convenient, because it allows one to employ a simple Ansatz for its longitudinal part. In particular, one may use the standard expression known form the studies of QED, namely [40]

$$
\widehat{\Gamma}^{\mu}\left(p_{1}, p_{2}, p_{3}\right)=\frac{A\left(p_{1}\right)+A\left(p_{2}\right)}{2} \gamma^{\mu}+\frac{\left(p_{1}-p_{2}\right)^{\mu}}{p_{1}^{2}-p_{2}^{2}}\left\{\left[A\left(p_{1}\right)-A\left(p_{2}\right)\right] \frac{\not p_{1}-\not p_{2}}{2}+\left[B\left(p_{1}\right)-B\left(p_{2}\right)\right]\right\} .
$$

The main advantage of this Ansatz is that it does not involve the so-called quark-ghost kernel, whose general structure is only partially known.

Of course, in the case of including various quark loops, corresponding to different quark flavors $N_{f}$, the term $\widehat{X}^{\mu v}(q)$ in Eq. (3.5) is replaced simply by the sum over all quark loops, i.e.,

$$
\widehat{X}^{\mu v}(q) \rightarrow \sum_{f} \widehat{X}_{f}^{\mu v}(q)
$$

Then, through the detailed analysis of [28] one reaches the conclusion that the unquenched gluon propagator $\Delta_{N_{f}}\left(q^{2}\right)$ may be expressed as a deviation from the quenched propagator $\Delta\left(q^{2}\right)$, namely (Euclidean space)

$$
\Delta_{N_{f}}\left(q^{2}\right)=\frac{\Delta\left(q^{2}\right)}{1+\left\{\widehat{X}\left(q^{2}\right)\left[1+G\left(q^{2}\right)\right]^{-2}+\lambda^{2}\left(q^{2}\right)\right\} \Delta\left(q^{2}\right)},
$$

where the quantity

$$
\lambda^{2}\left(q^{2}\right)=m_{N_{f}}^{2}\left(q^{2}\right)-m^{2}\left(q^{2}\right)
$$

measures the difference induced to the gluon mass due to the inclusion of quarks. In particular, $m_{N_{f}}^{2}\left(q^{2}\right)$ is to be obtained from Eq. (2.1) by implementing on its rhs the substitution $\Delta\left(q^{2}\right) \rightarrow \Delta_{N_{f}}\left(q^{2}\right)$.

We emphasize that, as one can demonstrate using a special identity [28], the nonperturbative $\widehat{X}\left(q^{2}\right)$ vanishes at the origin, $\widehat{X}(0)=0$, exactly as it happens in perturbation theory. This formal property is captured clearly in the numerical evaluation of $\widehat{X}\left(q^{2}\right)$, shown in Fig. 5.

Thus, the inclusion of quark loops affects the value of the saturation point of the gluon propagator not directly through the presence of the $\widehat{X}\left(q^{2}\right)$, but rather indirectly through the generation of a non vanishing mass difference $\lambda^{2}\left(q^{2}\right)$.

The next step is to treat the mass equation Eq. (2.1) and the unquenching master formula of Eq. (3.9) as a coupled system, and determine simultaneously both $m_{N_{f}}^{2}$ and $\Delta_{N_{f}}\left(q^{2}\right)$. In doing that, the nonperturbative form of each quark propagator entering into $\widehat{X}\left(q^{2}\right)$ will be obtained from the 


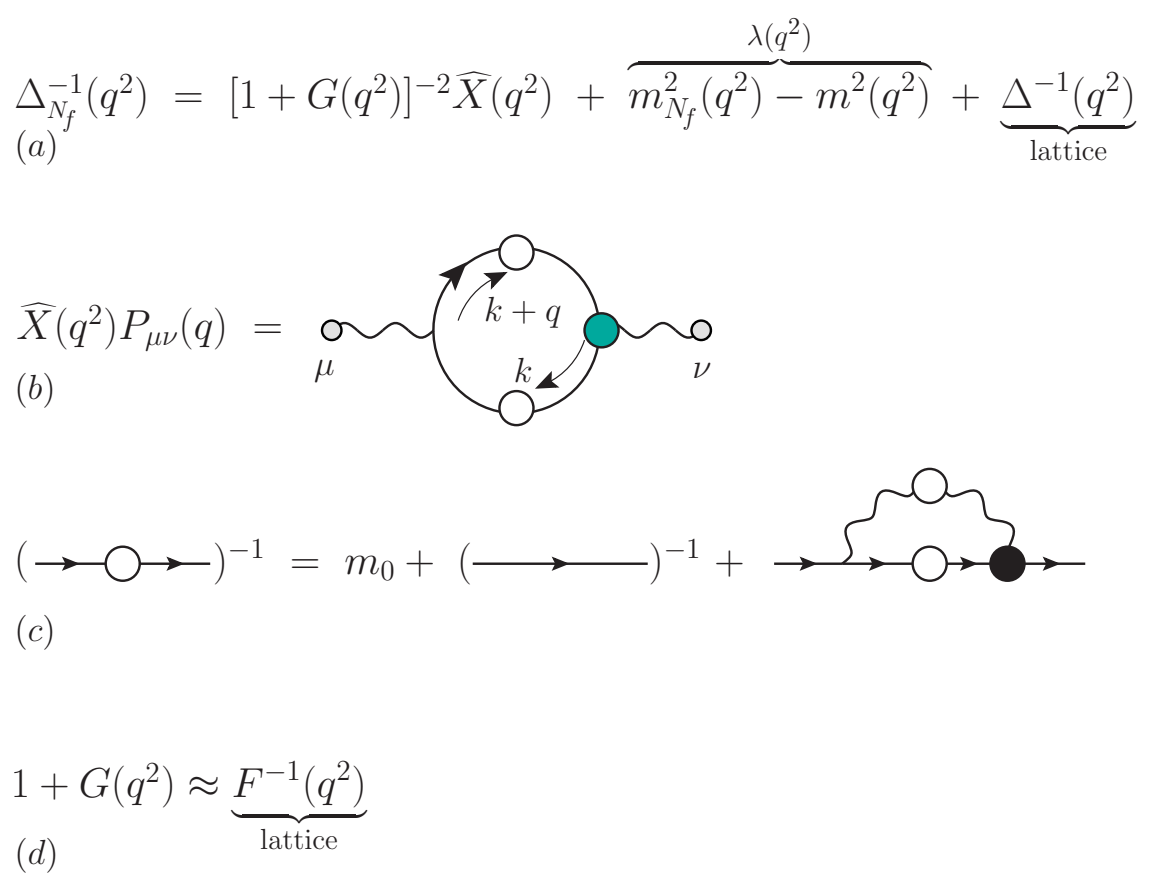

Figure 3: Schematic representation of the unquenched propagator (a), corresponding to Eq.(3.9), and some of the ingredients $[(b),(c)$, and $(d)]$ entering in it. In particular, $(b)$ represents the quark loop, which, in the approximation employed, is the only source of quark-dependence. The quark propagators entering in $(b)$ are solutions of the gap equation depicted in $(c)$, where $m_{0}$ denotes the appropriate current mass. Finally, the function $G\left(q^{2}\right)$ is obtained from the relation shown in $(d)$. The quantities obtained from the lattice are also indicated. Note that $\lambda^{2}\left(q^{2}\right)$ will be determined dynamically, once the mass equation is coupled to $(a)$.

standard gap equation [41], supplemented by an appropriate current mass term, in order to make contact with the lattice results of [19]. In this latter simulation, the gluon (and ghost) propagators have been evaluated from large volume configurations (up to $3^{3} \times 6\left[\mathrm{fm}^{4}\right]$ ), generated from a lattice action that included (twisted mass) fermions. Specifically, one employed two light degenerate quarks $\left(N_{f}=2\right)$, with a current mass ranging from 20 to $50 \mathrm{MeV}$, or two light and two heavy quarks $\left(N_{f}=2+1+1\right)$, with a strange (charm) quark current mass roughly set to $95 \mathrm{MeV}(1.51 \mathrm{GeV})$. Thus, effectively, one ends up dealing with the rather extended set of equations depicted in Fig. 3.

\section{Numerical results}

The system of SDEs that we consider is composed of Eqs. (2.1), (3.6) and (3.9), supplemented by the quark gap equation. The initial condition is provided by the quenched $S U(3)$ gluon propagator and ghost dressing function obtained in the lattice simulations of [7], which will be also used to determine the initial values of the form factors $A(p)$ and $\mathscr{M}(p)$. All calculations will be performed using propagators renormalized at $\mu=4.3 \mathrm{GeV}$.

The algorithm that we employ consists of the following main steps.

( $i$ ) We use the quenched propagator as an input of the first iterative step, and determine the quark form factors $A(p)$ and $\mathscr{M}(p)$ by solving the quark gap equation (see the results in Fig. 4). 

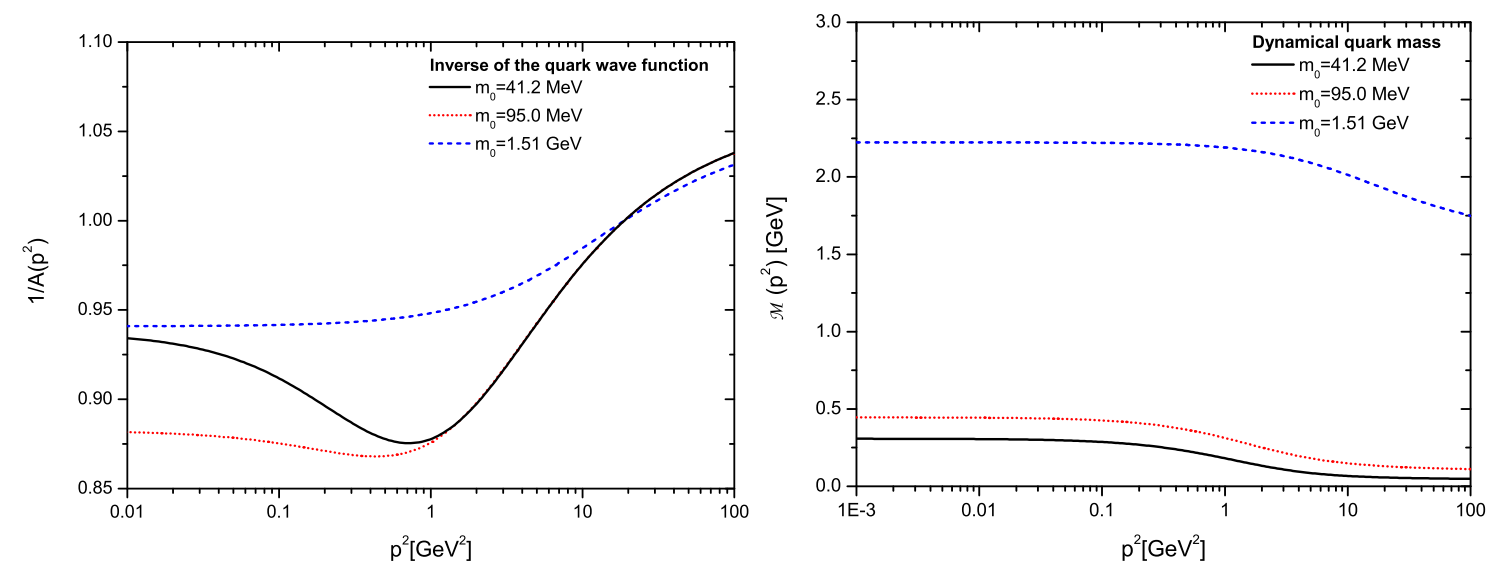

Figure 4: The inverse of the quark wave-functions (left panel), and the dynamical quark masses (right panel), obtained from the quark gap equation for three different values of the current mass: $m_{0}=41.2 \mathrm{MeV}$ (black, continuous), $m_{0}=95 \mathrm{MeV}$ (red, dotted) and $m_{0}=1.51 \mathrm{GeV}$ (blue, dashed).

(ii) $A(p)$ and $\mathscr{M}(p)$ are then substituted into Eq. (3.6), and the corresponding value of the quark loop diagram $\widehat{X}\left(q^{2}\right)$ is evaluated (results in Fig. 5).

(iii) The preliminary form of $\Delta_{N_{f}}\left(q^{2}\right)$ is determined from (3.9), employing initially $\lambda^{2}\left(q^{2}\right)=0$, with the quenched mass $m^{2}\left(q^{2}\right)$ obtained from the solution of the mass equation (2.1) corresponding to the quenched lattice propagator.

(iv) The unquenched propagator $\Delta_{N_{f}}\left(q^{2}\right)$ of the previous step is substituted into the mass equation (2.1) in order to determine the associated unquenched dynamical gluon mass $m_{N_{f}}^{2}\left(q^{2}\right)$, and therefore the corresponding $\lambda^{2}\left(q^{2}\right)$.

(v) At this point the latter quantity is inserted back into the master equation (3.9), and the loop starts again, until convergence, determined by the stability of the quantities involved, has been reached.

In Fig. 6 we present the central result of this analysis. In particular, we plot the propagators obtained when convergence of the above mentioned iteration procedure has been reached, and compare them with the corresponding unquenched lattice data, recently reported in [19]. We observe a rather good agreement between our theoretical predictions and the lattice computation for both values of $N_{f}$, for the available range of physical momenta. A notable exception to this fair coincidence between curves is the saturation point of the $N_{f}=2+1+1$ case; specifically, the value obtained from our SDE analysis is $20 \%$ higher than that found in lattice simulations.

Similar conclusions can be drawn by observing the plot corresponding to the gluon dressing functions, $q^{2} \Delta_{N_{f}}\left(q^{2}\right)$, shown in Fig. 7: while one has an excellent agreement in the case of two degenerate light quarks, when two heavier quarks are added the SDE solution tends to mildly overestimate the amplitude of the characteristic peak,x located in the intermediate momentum region.

The corresponding dynamical gluon masses, $m_{2}^{2}\left(q^{2}\right)$ and $m_{2+1+1}^{2}\left(q^{2}\right)$, are shown in Fig. 8; for comparison, we also plot the quenched solution, $m^{2}\left(q^{2}\right)$, obtained from Eq. (2.1) when the quenched lattice propagators of [7] are used as input. In particular, the corresponding saturation points give $m_{2}(0)=413 \mathrm{MeV}$ and $m_{2+1+1}(0)=425 \mathrm{MeV}$ (at $\mu=4.3 \mathrm{GeV}$ ), which should be compared with the value $m(0)=376 \mathrm{MeV}$ found in the quenched case. The results captured 


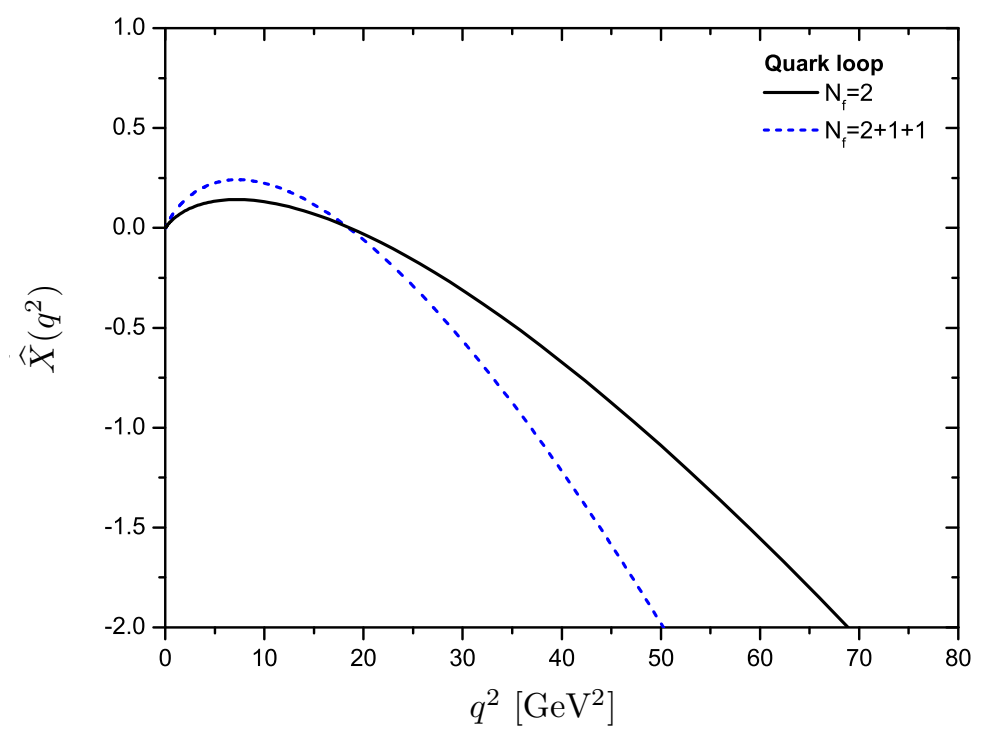

Figure 5: The full nonperturbative quark loop contribution $\widehat{X}\left(q^{2}\right)$ for the two cases $N_{f}=2$ (black, continuous) and $N_{f}=2+1+1$ (blue, dashed).

in Fig. 8 are particularly important, because they demonstrate clearly that the mass generation mechanism established for pure Yang-Mills continues to operate in QCD-like circumstances.

A this point it seems reasonable to think that the observed deviation between our results and the lattice signals a mild violation of one of the assumptions underlying the derivation of the unquenching formula (3.9). In particular, it is natural to expect that our main operating hypothesis, namely that the quark-loop contributions constitute a "perturbation" of the quenched propagator, becomes progressively less accurate as the number of active flavors increases. It is therefore possible that from $N_{f}>2$ onward we begin to perceive the onset of additional effects, not captured by (3.9).

In particular, the "lowest order unquenching" assumed here includes explicitly only the contribution of the quark loop $\widehat{X}\left(q^{2}\right)$, keeping all other quantities unquenched. This is reflected clearly at the level of the master formula Eq. (3.9), where the quantity $1+G\left(q^{2}\right)$ (or, equivalently, $F^{-1}\left(q^{2}\right)$, by virtue of Eq. (2.5)) assumes its quenched form, obtained from [7]. Moreover, the computation of $\widehat{X}\left(q^{2}\right)$ [see Fig. 3] uses as input the quark propagator obtained from the gap equation, which, in turn, depends on both the gluon propagator and the ghost dressing function; again, the quenched forms of [7] were employed. Finally, the strength of the gauge coupling $g$ also depends on the number of flavors; in the present analysis we have used its value when $N_{f}=0$ (MOM [42]). In order to improve this analysis, and eventually reach a better agreement with the lattice, one could gradually introduce quark effects into some of the aforementioned (quenched) ingredients. For example, one could envisage the possibility of using unquenched instead of quenched data for the ghost dressing function $F\left(q^{2}\right)$, obtained from the lattice analysis of [19]. Given that this quantity enters both in the master formula and the gap equation, its overall effect may be appreciable. In addition, the increase in the value of the gauge coupling produced by the inclusion of quark flavors may modify our predictions in the direction of the lattice data.

Furthermore, an additional theoretical uncertainty originates from the approximate (pertur- 

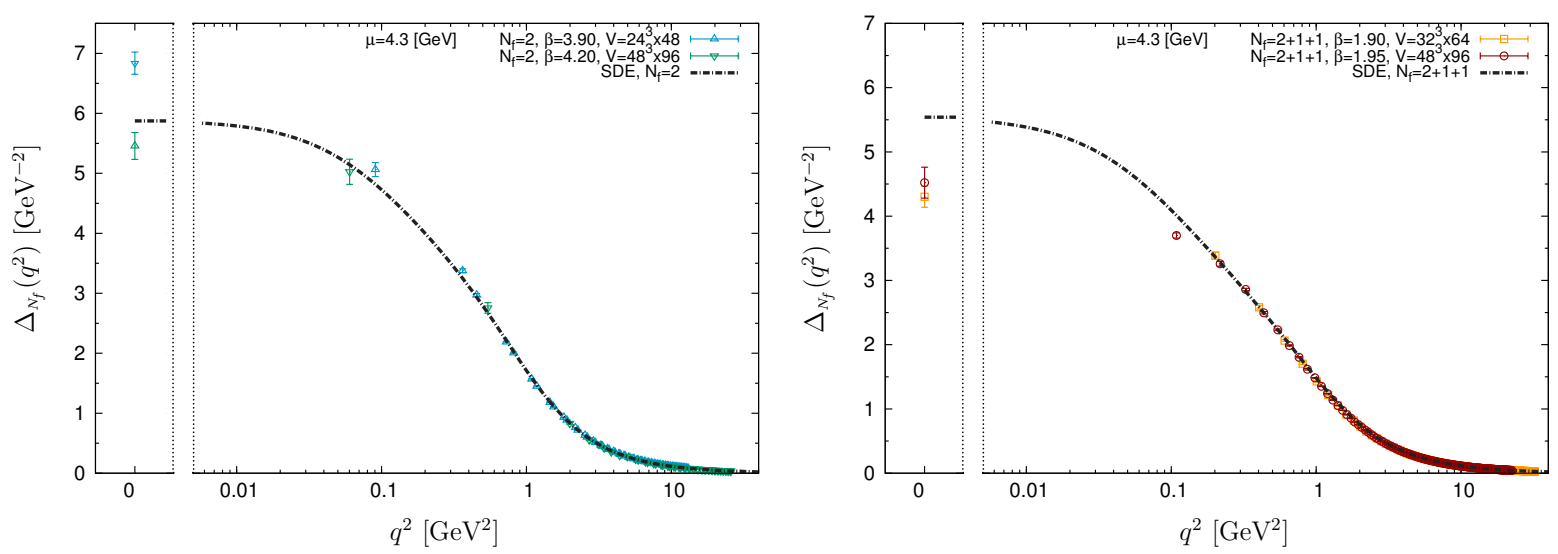

Figure 6: The unquenched gluon propagators obtained from our analysis, for $N_{f}=2$ (left panel) and $N_{f}=2+1+1$ (right panel), compared with the lattice data of [19] for the same cases.
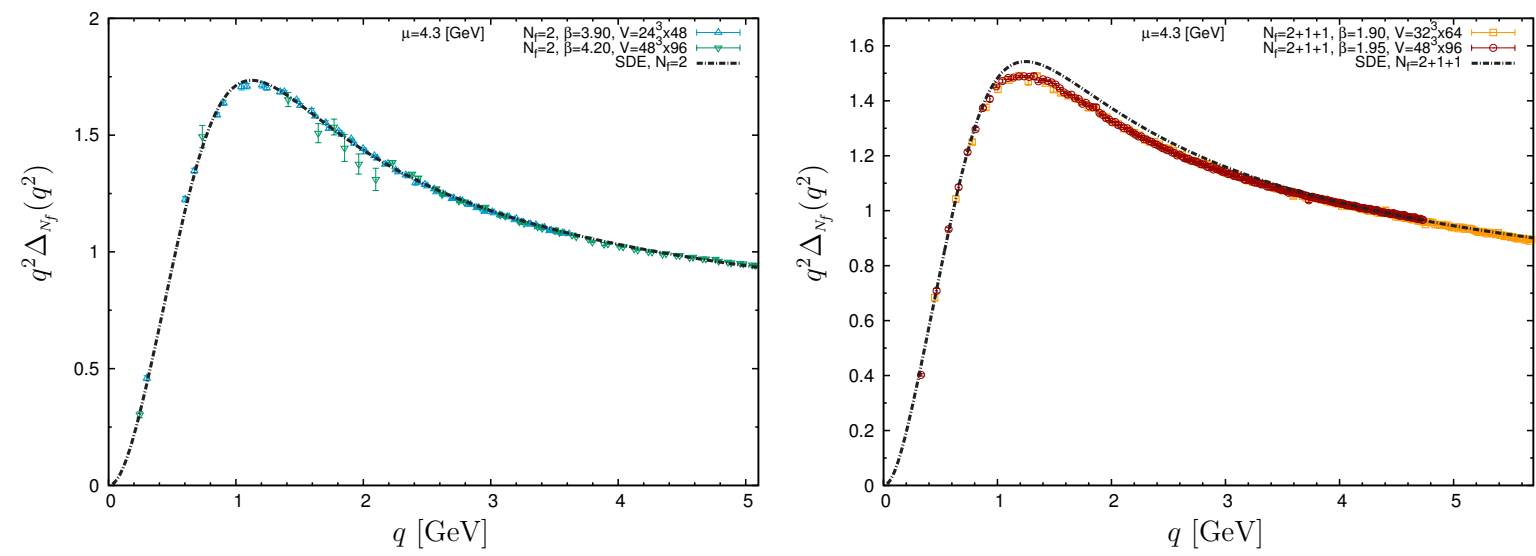

Figure 7: The unquenched gluon dressing functions obtained from our analysis, for $N_{f}=2$ (left panel) and $N_{f}=2+1+1$ (right panel), compared with the lattice data of [19] for the same cases.

bative) treatment of the quantity $Y\left(k^{2}\right)$. In particular, the parameter $C$ may only model, to some extent, unknown contributions that display a logarithmic momentum dependence, as in Eq. (2.6), but cannot account for terms with a different functional form. Moreover, the use of an Ansatz for the vertex $\widehat{\Gamma}_{\mu}$ entering into the definition of $\widehat{X}\left(q^{2}\right)$ may induce further error, due to the fact that its transverse (automatically conserved part) is in general undetermined.

\section{Conclusions}

In this presentation, by employing a methodology relying mainly on the SDEs that describe the gluon two-point sector within the PT-BFM framework, we studied in quantitative detail how the inclusion of dynamical quarks affects the generation of the momentum-dependent gluon mass, in the Landau gauge. Our main conclusion is that the gluon propagator continues to saturate in the infrared, due to the dynamical generation of a gluon mass. In fact, the analysis suggests that the gluon mass becomes heavier as the number of active quark families increases. It would be 


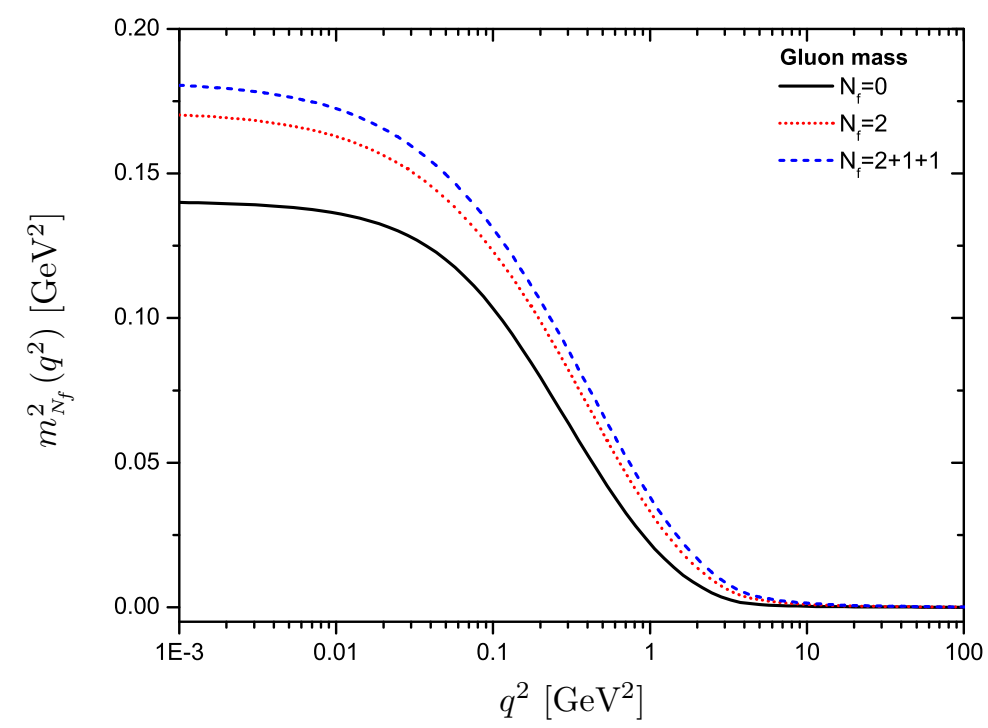

Figure 8: Solution of the mass equation yielding the dynamically generated gluon mass for $N_{f}=2$ (red dotted line) and $N_{f}=2+1+1$ (blue dashed line). In the deep infrared one has $m_{2}(0)=413 \mathrm{MeV}$, and $m_{2+1+1}(0)=425 \mathrm{MeV}$. For comparison we also show the quenched gluon mass (black continuous line) obtained from the quenched lattice propagator, in which case $m(0)=376 \mathrm{MeV}$.

interesting to study the possible limitations of this picture, and determine whether there is a critical number of quark families, past which the gluon generation mechanism clashes with the quarkinduced dynamics $[43,44]$.

\section{Acknowledgements:}

I would like to thank the ECT* for making this workshop possible. This research is supported by the Spanish MEYC under grant FPA2011-23596.

\section{References}

[1] A. C. Aguilar and J. Papavassiliou, JHEP 0612, 012 (2006).

[2] A. C. Aguilar, D. Binosi and J. Papavassiliou, Phys. Rev. D 78, 025010 (2008).

[3] P. Boucaud, J. P. Leroy, A. Le Yaouanc, J. Micheli, O. Pene and J. Rodriguez-Quintero, JHEP 0806, 099 (2008).

[4] R. Alkofer and L. von Smekal, Phys. Rept. 353, 281 (2001).

[5] M. Q. Huber and L. von Smekal, JHEP 1304, 149 (2013).

[6] A. Cucchieri and T. Mendes, PoS LAT2007, 297 (2007).

[7] I. L. Bogolubsky, E. M. Ilgenfritz, M. Muller-Preussker and A. Sternbeck, Phys. Lett. B 676, 69 (2009).

[8] J. M. Pawlowski, Annals Phys. 322, 2831 (2007).

[9] A. P. Szczepaniak and H. Reinhardt, Phys. Rev. D 84, 056011 (2011). 
[10] D. R. Campagnari and H. Reinhardt, Phys. Lett. B 707, 216 (2012).

[11] M. Quandt, H. Reinhardt and J. Heffner, arXiv:1310.5950 [hep-th].

[12] A. C. Aguilar, D. Ibanez, V. Mathieu and J. Papavassiliou, Phys. Rev. D 85, 014018 (2012).

[13] D. Binosi, D. Ibanez and J. Papavassiliou, Phys. Rev. D 86, 085033 (2012).

[14] C. D. Roberts and A. G. Williams, Prog. Part. Nucl. Phys. 33, 477 (1994).

[15] J. M. Cornwall, Phys. Rev. D 26, 1453 (1982).

[16] O. Philipsen, Nucl. Phys. B 628, 167 (2002).

[17] V. P. Nair, Phys. Rev. D 88, 105027 (2013).

[18] A. C. Aguilar, D. Binosi, D. Ibanez and J. Papavassiliou, arXiv:1312.1212 [hep-ph].

[19] A. Ayala, A. Bashir, D. Binosi, M. Cristoforetti and J. Rodriguez-Quintero, Phys. Rev. D 86, 074512 (2012).

[20] J. M. Cornwall and J. Papavassiliou, Phys. Rev. D 40, 3474 (1989).

[21] A. Pilaftsis, Nucl. Phys. B 487, 467 (1997).

[22] D. Binosi and J. Papavassiliou, Phys. Rept. 479, 1 (2009)

[23] L. F. Abbott, Nucl. Phys. B 185, 189 (1981).

[24] A. Bashir, A. Raya and J. Rodriguez-Quintero, arXiv:1401.1447 [hep-ph], these Proceedings.

[25] C. S. Fischer and R. Alkofer, Phys. Rev. D 67, 094020 (2003).

[26] C. S. Fischer, P. Watson and W. Cassing, Phys. Rev. D 72, 094025 (2005).

[27] A. C. Aguilar, D. Binosi and J. Papavassiliou, Phys. Rev. D 88, 074010 (2013).

[28] A. C. Aguilar, D. Binosi and J. Papavassiliou, Phys. Rev. D 86, 014032 (2012).

[29] J. S. Schwinger, Phys. Rev. 125, 397 (1962).

[30] J. S. Schwinger, Phys. Rev. 128, 2425 (1962).

[31] R. Jackiw and K. Johnson, Phys. Rev. D 8, 2386 (1973).

[32] J. M. Cornwall and R. E. Norton, Phys. Rev. D 83338 (1973).

[33] E. Eichten and F. Feinberg, Phys. Rev. D 10, 3254 (1974).

[34] E. C. Poggio, E. Tomboulis and S. H. Tye, Phys. Rev. D 11, 2839 (1975).

[35] D. Ibanez and J. Papavassiliou, Phys. Rev. D 87, no. 3, 034008 (2013).

[36] P. A. Grassi, T. Hurth and M. Steinhauser, Annals Phys. 288, 197 (2001).

[37] P. A. Grassi, T. Hurth and A. Quadri, Phys. Rev. D 70, 105014 (2004).

[38] A. Cucchieri, A. Maas and T. Mendes, Phys. Rev. D 77, 094510 (2008).

[39] A. C. Aguilar, D. Binosi and J. Papavassiliou, arXiv:1401.3631 [hep-ph].

[40] D. C. Curtis and M. R. Pennington, Phys. Rev. D 48, 4933 (1993).

[41] A. C. Aguilar and J. Papavassiliou, Phys. Rev. D 83, 014013 (2011).

[42] P. Boucaud, F. De Soto, J. P. Leroy, A. Le Yaouanc, J. Micheli, O. Pene and J. Rodriguez-Quintero, Phys. Rev. D 79, 014508 (2009).

[43] X. Cheng and E. T. Tomboulis, PoS QCD -TNT-II, 046 (2011).

[44] E. T. Tomboulis, Phys. Rev. D 87, 034513 (2013). 